\title{
Role of Dispersion Forces in the Structure of Graphene Monolayers on Ru Surfaces
}

\author{
D. Stradi, ${ }^{1,2}$ S. Barja, ${ }^{3,2}$ C. Díaz, ${ }^{1}$ M. Garnica, ${ }^{3,2}$ B. Borca,${ }^{3}$ J. J. Hinarejos, ${ }^{3}$ D. Sánchez-Portal,,${ }^{4,5}$ M. Alcamí, ${ }^{1}$ \\ A. Arnau, ${ }^{4,5,6}$ A. L. Vázquez de Parga, ${ }^{3,2}$ R. Miranda, ${ }^{3,2}$ and F. Martín ${ }^{1,2, *}$ \\ ${ }^{1}$ Departamento de Química, Módulo 13, Universidad Autónoma de Madrid, 28049 Madrid, Spain \\ ${ }^{2}$ Instituto Madrileño de Estudios Avanzados en Nanociencia (IMDEA-Nanociencia), Cantoblanco, 28049 Madrid, Spain \\ ${ }^{3}$ Dep. Física de la Materia Condensada, Universidad Autónoma de Madrid, 28049 Madrid, Spain \\ ${ }^{4}$ Materials Physics Center (CSIC-UPV/EHU), Paseo Manuel de Lardizábal 5, 20018 San Sebastián, Spain \\ ${ }^{5}$ Donostia International Physics Centre (DIPC), Paseo Manuel Lardizábal 4, 20018 San Sebastián, Spain \\ ${ }^{6}$ Dep. Física de Materiales (UPV/EHU), Facultad de Química, Apartado 1072, 20080 San Sebastián, Spain
}

(Received 3 November 2010; published 5 May 2011)

Elaborate density functional theory (DFT) calculations that include the effect of van der Waals (vdW) interactions have been carried out for graphene epitaxially grown on $\mathrm{Ru}(0001)$. The calculations predict a reduction of structural corrugation in the observed moiré pattern of about $25 \%(\sim 0.4 \AA)$ with respect to DFT calculations without vdW corrections. The simulated STM topographies are close to the experimental ones in a wide range of bias voltage around the Fermi level.

DOI: 10.1103/PhysRevLett.106.186102

The exceptional electronic properties of perfect twodimensional graphene, such as its high electron mobility or its anomalous quantum Hall effect [1], makes it a promising material for applications in microelectronics and sensing. In practice, synthesis of graphene can be achieved by epitaxial growth of carbon monolayers on transition metals [2-5]. This has stimulated a lot of experimental work on different metal substrates [4,6-14], which has already led to the production of relatively large domains (a crucial aspect to reproduce the properties of infinitely planar graphene). Nevertheless, in most cases, the presence of the substrate leads to modifications of the graphene morphology. One of these is the appearance of moiré patterns [3], as it is the case for graphene grown on $\mathrm{Ru}(0001)(\mathrm{G} / \mathrm{Ru}$ for short). The presence of moiré patterns not only implies changes in the graphene morphology but also in its electron density, which is no longer uniform [4]. This non uniform electron density is responsible for, e.g., the formation of quantum dots $[15,16]$ or the selective deposition of organic molecules on well defined regions of the graphene sheet $[17,18]$.

In spite of the efforts to determine the structural corrugation of the moiré observed in $\mathrm{G} / \mathrm{Ru}$, the actual value of such corrugation is still a subject of controversy. Low energy electron diffraction (LEED) experiments give a corrugation of $1.5 \AA$ [19], while surface x-ray diffraction (SXRD) measurements suggest two possible values, 0.82 and $1.5 \AA[6,20]$. In scanning tunneling microscopy (STM) measurements, the apparent corrugation of the moiré ripples decreases from 1.1 to $0.5 \AA$ when the tunneling bias voltage goes from $-0.8 \mathrm{~V}$ to $+0.8 \mathrm{~V}$ [21]. Helium atom scattering (HAS) experiments, that are sensitive to the surface total charge corrugation, give $0.15-0.4 \AA$ [22] On the theoretical side, density functional theory (DFT) calculations [19,23-26], in which the $\mathrm{C}$ and $\mathrm{Ru}$ high
PACS numbers: 73.22.Pr, 68.37.Ef, 68.55.-a, 71.15.Mb

symmetry directions are aligned but the unit cell is large enough to account for the moiré pattern, predict a corrugation in the range $\sim 1.5-1.7 \AA$ [23-25].

In the latter calculations, the effect of van der Waals (vdW) or dispersion forces could not be included. However, there is strong evidence that these forces play a crucial role in the adsorption of aromatic molecules on metal surfaces [27-31], leading, in comparison with nonvdW DFT calculations, to a significant increase of adsorption energies [30] and, what is even more important, to significant geometric distortions of both the adsorbed molecule and the metal substrate [32]. In many respects, graphene can be considered as an extended aromatic system due to the presence of a $\pi$ electronic cloud above and below the graphene plane. Therefore, it is worth studying if $\mathrm{vdW}$ interactions play a similar important role in the structure of graphene lying on top of a metal substrate.

Existing attempts to include $\mathrm{vdW}$ interactions in G/metal systems [33,34] are limited to models in which the graphene sheet is planar and forced to follow the periodicity of the metal substrate by using a $1 \times 1$ unit cell. In this Letter we show that, when vdW interactions are included in DFT calculations in which the size of the unit cell is large enough to account for the moiré pattern observed in STM images of $\mathrm{G} / \mathrm{Ru}$, a reduction of corrugation of $\sim 0.4 \AA$ with respect to DFT calculations without $\mathrm{vdW}$ is observed. The calculations predict a variation of the apparent corrugation with tunneling bias voltage in qualitative agreement with that observed in STM experiments in the range from -2.0 to $+2.0 \mathrm{~V}$. All DFT calculations were performed using the VASP code $[35,36]$. As in Ref. [19], the Perdew, Burke, and Ernzerhof (PBE) [37,38] generalized gradient approximation (GGA) functional was used. The effect of vdW interactions was taken into account by using the empirical correction scheme of Grimme 
(DFT + D/PBE) [39], as implemented by Bucko et al [40] for periodic systems. Standard values for the dispersion coefficients $C_{6}\left(1.75\right.$ and $24.67 \mathrm{~J} \mathrm{~nm}^{6} \mathrm{~mol}^{-1}$, for $\mathrm{C}$ and $\mathrm{Ru}$, respectively), vdW radii (1.452 and $1.639 \AA$ ), cutoff radius for pair interactions (30.0 $\mathrm{A}$ ), PBE global scaling factor (0.75), and damping factor (20.0) have been used. These values have been shown to give geometries in good agreement with experiment $[30,32,39]$. The $\mathrm{G} / \mathrm{Ru}$ surface was modeled within the supercell approach considering a single $11 \times 11$ graphene sheet over three $10 \times 10$ Ru layers and a vacuum region of $10.2 \AA$. This supercell presents the minimum lattice mismatch between the graphene lattice and the $\mathrm{Ru}(0001)$ surface. This choice differs from the $25 \times 25$ one proposed by SXRD experiments [6] to account for the slight rotation of the graphene overlayer with respect to the Ru topmost layer [22] (calculations for the latter cell still remain prohibitively expensive). The $11 \times 11$ unit cell was used in Ref. [26] (without including $\mathrm{vdW}$ corrections), where it was shown to yield a binding energy and a corrugation similar to those of the slightly larger $12 \times 12$ and $13 \times 13$ unit cells. The reported values for the corrugation are $1.62 \AA$ [26] and $1.75 \AA$ [25] $(11 \times 11), 1.50 \AA[24], 1.67 \AA[25], 1.51 \AA$ [26] and $1.50 \AA$ [19] $(12 \times 12)$, and $1.44 \AA$ [26] and $1.59 \AA$ [19] $(13 \times 13)$. In the present work, the entire graphene sheet and the $\mathrm{Ru}$ topmost layer were allowed to relax during geometry optimization. Both DFT/PBE and DFT + $\mathrm{D} / \mathrm{PBE}$ calculations were performed by using a convergence criterion for residual forces acting on the atoms of $0.01 \mathrm{eV} / \AA$, i.e., three, five and 10 times smaller than in $[19,25,26]$, respectively. Valence electrons of both $C$ and $\mathrm{Ru}$ as well as $\mathrm{Ru}$ semi-core $p$ electrons not taken into account in previous theoretical works were included. As in previous works, the cutoff of the plane-wave expansion was set to a kinetic energy of $400 \mathrm{eV}$. The ionic cores were described by the projector augmented wave (PAW) method [41]. Taking into account the large size of the supercell employed, the Brillouin zone sampling was restricted to the $\Gamma$ point, using a Methfessel-Paxton smearing [42] of $0.2 \mathrm{eV}$. The appropriateness of such a sampling was checked by performing systematic calculations for stretched planar graphene lying over three Ru layers by using a $1 \times 1$ unit cell. In the latter calculations, convergence was achieved for a significantly less dense $k$ sampling than that used in the actual calculations (i.e., less dense than the sampling corresponding to a Brillouin zone close in size to that associated with the large unit cell). Finally, constant current STM images at different bias voltages $V_{s}$ have been evaluated by using the TersoffHamann approach [43] on an electron density contour of $1.69 \times 10^{-4} \AA^{-3}$, which approximately corresponds to typical tunneling conditions of about $1 \mathrm{nA}$ and $1 \mathrm{~V}$ [44].

The experiments were done in a ultra high vacuum (UHV) chamber equipped with a Low Temperature STM working between 4.6 and $300 \mathrm{~K}$ (see [15]). The STM tips were cleaned by sputtering and annealing in UHV [45].
Figure 1(a) shows the calculated ground state geometry. The average errors in the vertical displacements due to (i) the criterion used to converge the forces or (ii) a poor representation of the $\mathrm{vdW}$ interaction for atoms lying below the second topmost $\mathrm{Ru}$ layer are 0.015 and $0.001 \AA$, respectively. The ripple height is $1.195 \AA$, which is $0.42,0.55$, and $0.40 \AA$ smaller than that obtained in $[25,26]$ here, respectively, without including vdW corrections for the same unit cell. Therefore, the substantial reduction in the calculated corrugation is due to the strong $\mathrm{vdW}$ interaction between the graphene sheet and the Ru substrate. This interpretation is further supported by the results shown in Fig. 1(b), where it can be seen that, in the absence of vdW interactions, forces of the order of $0.1 \mathrm{eV} / \AA$ act on the ripple $\mathrm{C}$ atoms in the sense of increasing graphene corrugation. When the $\mathrm{vdW}$ interaction is taken into account, the residual forces (of less than $0.01 \mathrm{eV} / \AA$ ) $)$ either lie in the $x y$ plane or point towards the metal surface. The calculated corrugation lies in between the values proposed in recent LEED $(1.53 \pm 0.2 \AA$ [19] $)$

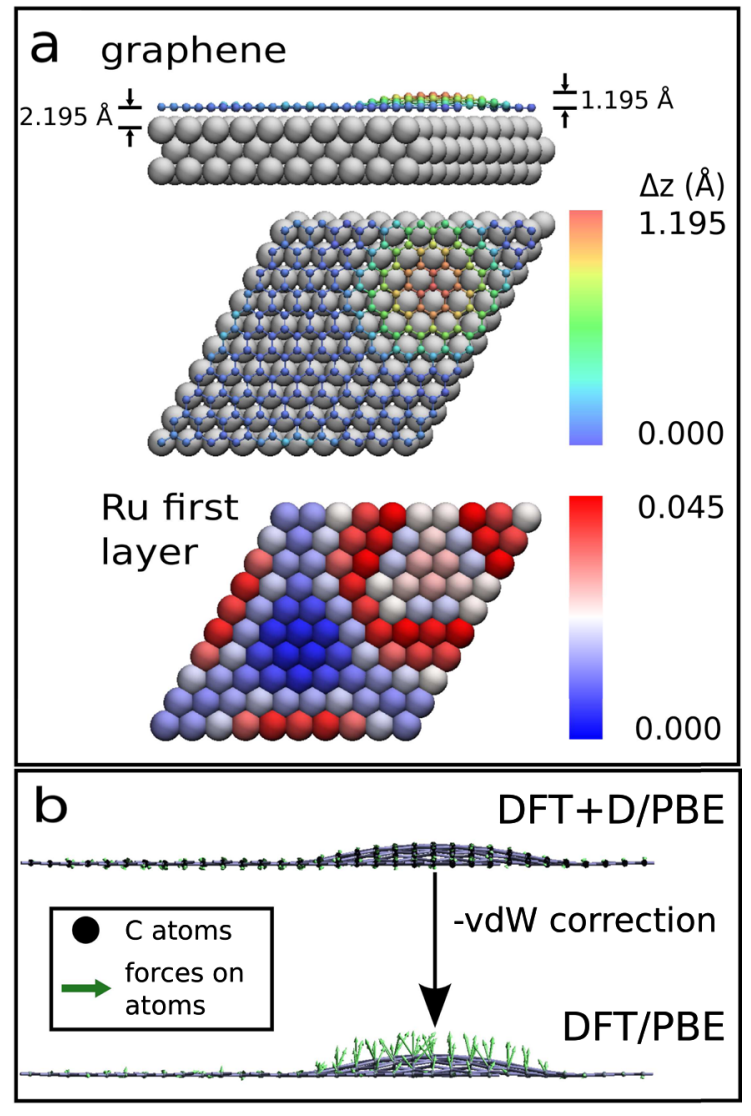

FIG. 1 (color online). (a) Top: calculated ground state geometry of graphene $/ \mathrm{Ru}(0001)$ with $(11 \times 11) /(10 \times 10)$ periodicity. $\mathrm{C}$ atoms of the graphene monolayer are printed in different colors depending on their relative height (the lowest $\mathrm{C}$ atom is set to $\Delta z=0.000 \AA$ ), while $\mathrm{Ru}$ atoms are printed in grey. Bottom: reconstruction of the $\mathrm{Ru}$ topmost layer. (b) Residual forces (arrows) acting on the $\mathrm{C}$ atoms of the graphene layer at the $\mathrm{DFT}+\mathrm{D} / \mathrm{PBE}$ level (top) and when the correction due to dispersion forces is removed (bottom). 
and SXRD (0.82 $\pm 0.15 \AA$ [20]) experiments and is higher than that inferred from HAS [22]. The reason for the latter discrepancy is not known. Interestingly, the minimum Ru$\mathrm{C}$ distance is only $0.105,0.035$, and $0.038 \AA$ smaller than that obtained without vdW corrections in $[25,26]$ and the present work, respectively, for the same unit cell. This relatively less important role of $\mathrm{vdW}$ interactions in the moiré valleys is the consequence of the rather strong covalent interaction between $\mathrm{C}$ and $\mathrm{Ru}$ atoms in this region; in contrast, the covalent character is very weak in the ripples.

Another interesting result is that, in agreement with the LEED measurements reported in [19], the buckling of the $\mathrm{Ru}$ topmost layer approximately follows that of the graphene layer; i.e., in general, $\mathrm{Ru}$ atoms below ripples lie higher than those below valleys. This subtle effect is not well reproduced by existing non-vdW DFT calculations, in which several $\mathrm{Ru}$ atoms lying just below the top of the ripple lie in the same plane as those lying below the center of the valleys. Nevertheless, the magnitude of the $\mathrm{Ru}$ displacements is smaller than suggested by LEED [19]. The vdW correction also leads to a substantial increase of the adsorption energy, from $27 \mathrm{meV} / \mathrm{C}$-atom (non-vdW calculation) to $206 \mathrm{meV} / \mathrm{C}$-atom. The former value is larger than that reported in previous work (11 [25] and $9 \mathrm{meV} / \mathrm{C}$-atom [26]) due to inclusion of Ru $p$ electrons in the present calculations.

The calculated STM topographies are in good agreement with the measured ones [4] in the range of $V_{s}$ from -2.0 to +2.0 V. As illustration, Figs. 2(a) and 2(b) show a comparison for $V_{s}=-1.0 \mathrm{~V}$. It can be seen that the periodic pattern in the STM image is well reproduced by theory. A more stringent test is to compare profiles of apparent height $(Z)$ along specific directions. Figures $2(\mathrm{c})$ and d show the results along the path shown in panels (a) and (b). The pattern of inverted muffin tins is reasonably reproduced by theory, especially when the latter takes into account the experimental resolution.

Along the same path, Fig. 3 shows the variation of the apparent corrugation, $\Delta Z=Z_{\max }-Z_{\min }$, with $V_{s}$ in the interval $[-2.0,+2.0] \mathrm{V}[46]$. The experimental results were obtained for different experimental conditions, i.e., different tips, different samples, tunneling currents between 0.1 and $1.0 \mathrm{nA}$, and sample temperatures between 4.6 and $300 \mathrm{~K}$. Apart from a slight overestimation of the experimental values by about $0.1-0.2 \AA$, the overall variation with the bias is well described by theory. Both theory and experiment predict that the apparent corrugation is $\sim 1.0 \AA$ for $V_{s} \leq 0$ and then decreases with $V_{s}$ for $V_{s} \geq 0$ down to $\sim 0.5 \AA\left(V_{s}=+2.0 \mathrm{~V}\right)$. Although the simulated STM images were calculated on a contour of $1.69 \times$ $10^{-4} \AA^{-3}$, similar results have been obtained by using neighboring contours compatible with typical STM currents and tip-surface distances. The apparent corrugation curve resulting from non-vdW calculations (see Fig. 3) lies significantly above the experimental data.

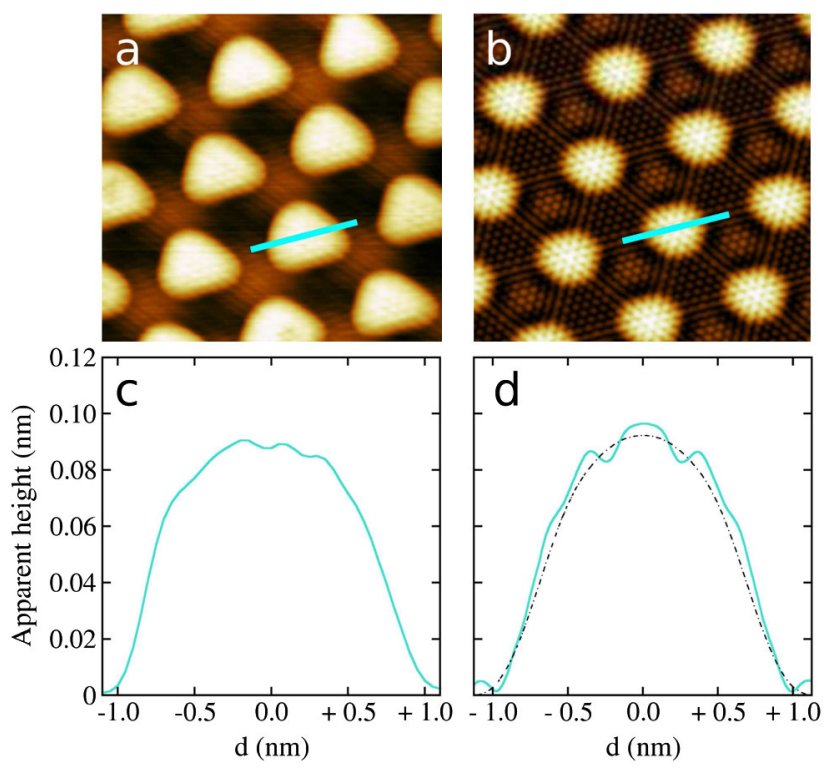

FIG. 2 (color online). (a) STM image of graphene/Ru(0001) for $V_{s}=-1.0 \mathrm{~V}$. (b) Simulated atomic resolution STM image for $V s=-1.0 \mathrm{~V}$ and an electron density contour of $1.69 \times$ $10^{-4} \AA^{-3}$. (c) and (d) show the apparent height profiles taken along the paths shown in (a) and (b), respectively, as a function of the distance $d$ from the beginning of the path. The dashed line has been obtained with Gaussian smearing.

The vdW corrections used in this work were developed and consistently benchmarked for molecules in vacuum [39]. For $\mathrm{G} / \mathrm{Ru}$, one could expect that the values of the vdW $C_{6}$ coefficients are smaller than in the gas phase due to screening and image-charge effects associated with the delocalized electronic environment. In some cases, the reduction might be as large as a factor of 2 [47]. To check the influence of these effects on the present

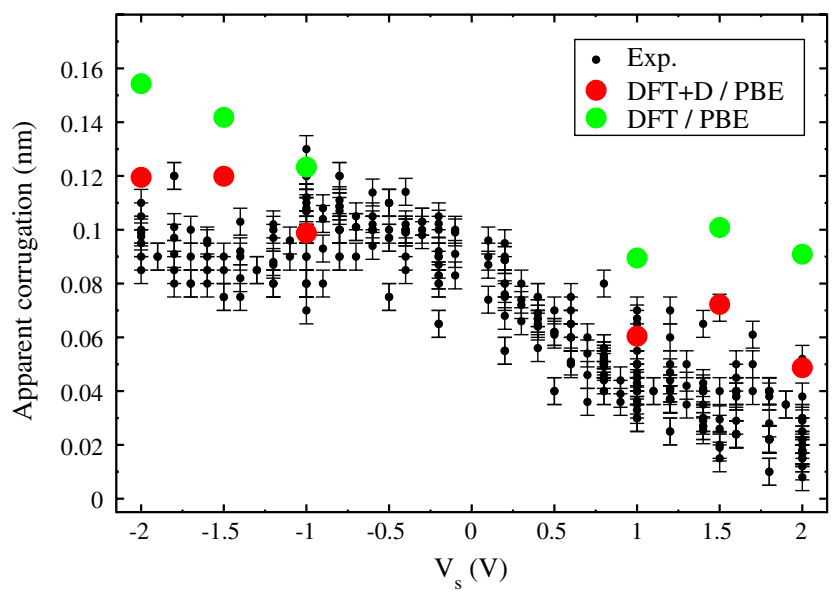

FIG. 3 (color online). Variation of the apparent corrugation with bias voltage along the path shown in Fig. 2. Black dots: experimental results obtained with many different experimental conditions (tip, tunnelling current, samples and temperature). Red dots: theoretical results with vdW correction. Green dots: idem without vdW corrections. Theoretical STM images were calculated on an electron density contour of $1.69 \times 10^{-4} \AA^{-3}$. 
results, we have repeated the calculations for the $11 \times 11$ cell by using a substantially smaller value for $\mathrm{Ru}, C_{6}=$ $15.562 \mathrm{~J} \mathrm{~nm}^{6} \mathrm{~mol}^{-1}$, and the value $C_{6}=1.65 \mathrm{~J} \mathrm{~nm}^{6} \mathrm{~mol}^{-1}$ for $\mathrm{C}$ that provides the best agreement with the experimental exfoliation energy (52 $\pm 5 \mathrm{meV}$ [48]) and interlayer distance (3.35 $\AA$ [49]) of graphite (50.29 meV and $3.25 \AA$, respectively); all the other parameters were maintained at the default values mentioned above (see [50] for a detailed analysis of the sensitivity of the results with the $C_{6}$ parameters). The corrugation of the ripple that results from these calculations is $1.239 \AA$, i.e., only $0.044 \AA$ higher than that reported above. The corrugation of the topmost $\mathrm{Ru}$ layer remains identical and the minimum distance between the $\mathrm{C}$ atoms of the graphene layer and the $\mathrm{Ru}$ atoms of the topmost Ru layer slightly varies from 2.195 to $2.242 \AA$. As expected, the adsorption energy is more affected and becomes significantly smaller: $0.163 \mathrm{eV} / \mathrm{C}$ atom. Since the value of $C_{6}$ used for $\mathrm{Ru}$ in these test calculations is probably far lower than what is reasonable to expect, one can safely conclude that the geometrical effects discussed above remain practically unchanged for other intermediate choices.

In conclusion, DFT $+\mathrm{D}$ calculations for a $11 \times 11$ unit cell show that vdW interactions between the graphene sheet and the $\mathrm{Ru}(0001)$ substrate are responsible for a reduction of $\sim 25 \%$ in the corrugation of the moiré pattern. The apparent corrugation calculated by including (excluding) vdW corrections is in good (poor) agreement with that observed in STM experiments. Its variation with bias voltage suggests a strong electronic effect.

We thank Mare Nostrum BSC and CCC-UAM for computer time. Work supported by the MICINN projects FIS2010-15127, FIS2010-18847, CTQ2010-17006, FIS2010-19609-C02-00, ACI2008-0777, 2010C-0725200, and CSD2007-00010, the CAM program NANOBIOMAGNET S2009/MAT1726, and the Gobierno Vasco-UPV/EHU project IT-366-07.

*fernando.martin@uam.es

[1] A. H. Castro-Neto et al., Rev. Mod. Phys. 81, 109 (2009).

[2] A. T. N'Diaye et al., Phys. Rev. Lett. 97, 215501 (2006).

[3] S. Marchini, S. Günther, and J. Wintterlin, Phys. Rev. B 76, 075429 (2007).

[4] A. L. Vázquez de Parga et al., Phys. Rev. Lett. 100, 056807 (2008).

[5] P. W. Sutter, J.-I. Flenge, and E. A. Sutter, Nature Mater. 7, 406 (2008).

[6] D. Martoccia et al., Phys. Rev. Lett. 101, 126102 (2008).

[7] J. Coraux et al., Nano Lett. 8, 565 (2008).

[8] A. B. Preobrajenski et al., Phys. Rev. B 78, 073401 (2008).

[9] P. Sutter et al., Nano Lett. 9, 2654 (2009).

[10] P. Lacovig et al., Phys. Rev. Lett. 103, 166101 (2009).

[11] J. Wintterlin and M.L. Bocquet, Surf. Sci. 603, 1841 (2009).
[12] Y. Pan et al., Adv. Mater. 21, 2777 (2009).

[13] M. Gao et al., Appl. Phys. Lett. 96, 053109 (2010).

[14] X. Li et al., Science 324, 1312 (2009).

[15] B. Borca et al., Phys. Rev. Lett. 105, 036804 (2010).

[16] H. G. Zhang et al., J. Phys. Condens. Matter 22, 302001 (2010).

[17] J. Mao et al., J. Am. Chem. Soc. 131, 14136 (2009).

[18] A. J. Pollard et al., Angew. Chem., Int. Ed. 49, 1794 (2010).

[19] W. Moritz et al., Phys. Rev. Lett. 104, 136102 (2010).

[20] D. Martoccia et al., New J. Phys. 12, 043028 (2010).

[21] A. L. Vázquez de Parga et al., Phys. Rev. Lett. 101, 099704 (2008).

[22] B. Borca et al., New J. Phys. 12, 093018 (2010).

[23] B. Wang et al., Phys. Chem. Chem. Phys. 10, 3530 (2008).

[24] B. Wang et al., Phys. Rev. Lett. 101, 099703 (2008).

[25] D. Jiang, M.-H. Du, and S. Dai, J. Chem. Phys. 130, 074705 (2009).

[26] B. Wang et al., New J. Phys. 12, 043041 (2010).

[27] P. Sony et al., Phys. Rev. Lett. 99, 176401 (2007).

[28] N. Atodiresei et al., Phys. Rev. Lett. 102, 136809 (2009).

[29] J. Brede et al., Phys. Rev. Lett. 105, 047204 (2010).

[30] G. Mercurio et al., Phys. Rev. Lett. 104, 036102 (2010).

[31] M. Mura et al., Phys. Chem. Chem. Phys. 12, 4759 (2010).

[32] E. R. McNellis et al., Phys. Chem. Chem. Phys. 12, 6404 (2010).

[33] M. Vanin et al., Phys. Rev. B 81, 081408 (2010).

[34] R. Brako et al., New J. Phys. 12, 113016 (2010).

[35] G. Kresse and J. Hafner, Phys. Rev. B 47, 558 (1993).

[36] G. Kresse and D. Joubert, Phys. Rev. B 59, 1758 (1999).

[37] J. P. Perdew, K. Burke, and M. Ernzerhof, Phys. Rev. Lett. 77, 3865 (1996).

[38] J. P. Perdew, K. Burke, and M. Ernzerhof, Phys. Rev. Lett. 78, 1396 (1997).

[39] S. Grimme, J. Comput. Chem. 27, 1787 (2006).

[40] T. Bücko et al., J. Phys. Chem. A 114, 11814 (2010).

[41] P. E. Blöchl, Phys. Rev. B 50, 17953 (1994).

[42] M. Methfessel and A. T. Paxton, Phys. Rev. B 40, 3616 (1989).

[43] J. Tersoff and D. R. Hamman, Phys. Rev. Lett. 50, 1998 (1983).

[44] W. A. Hofer, A. S. Foster, and A. L. Shluger, Rev. Mod. Phys. 75, 1287 (2003).

[45] A. L. Vázquez de Parga et al., Phys. Rev. Lett. 80, 357 (1998).

[46] Because of the artificial discretization introduced by the limited number of Ru layers in the slab, as well as by the $k$-point sampling used in the calculations, the simulated low bias apparent corrugations are not accurate enough and, therefore, they are not given.

[47] L. W. Bruch, M.W. Cole, and E. Zaremba, Physical Adsorption: Forces and Phenomena (Dover Publications, Mineol, NY, 2007).

[48] R. Zacharia, H. Ulbricht, and T. Hertel, Phys. Rev. B 69, 155406 (2004).

[49] Y. X. Zhao and I. L. Spain, Phys. Rev. B 40, 993 (1989).

[50] See supplemental material at http://link.aps.org/ supplemental/10.1103/PhysRevLett.106.186102. 